\title{
Determination of astrophysical parameters of open clusters using classical and semi-automated isochrone-fitting methods
}

\author{
Virginia Mello Alves, ${ }^{1}$ Eduardo Bica ${ }^{2}$ and Daniela Borges Pavani ${ }^{1}$ \\ ${ }^{1}$ Universidade Federal de Pelotas (UFPel), Brazil \\ ${ }^{2}$ Universidade Federal do Rio Grande do Sul (UFRGS), Brazil
}

\begin{abstract}
When we use optical isochrone-fitting solutions from the literature to 2MASS CMDs, they are often not the best solutions in the infrared domain. We analysed 10 open clusters with 2MASs, nine of them previously studied with optical photometry (NGC 1245, NGC 1342, NGC 1502, NGC 2104, NGC 2204, NGC 2243, NGC 2281, NGC 6709 and NGC 744) and one using integrated spectroscopy (BH 132). The study involved the classical (by eye) and a semiautomated method of isochrone fitting. We used the solutions of the first method as input for the second, looking for refined solutions. The semi-automated method uses a synthetic colormagnitude diagram (CMD), based on different Padova isochrones, to compare with the observed CMDs by means of likelihood statistics. The derived astrophysical parameters are age, distance and reddening values. The present results show better fits than those implied by the optical values. We also show that the semi-automated method decreases the parameter uncertainties.
\end{abstract}

Keywords. open clusters and associations: general, infrared: stars

The full poster (in pdf format) is available at http://www . astro.iag.usp.br/〜iaus266/Posters/pAlves.pdf. 